\title{
MicroRNA-155 targets myosin light chain kinase to inhibit the migration of human bone marrow-derived mesenchymal stem cells
}

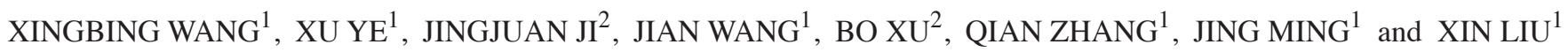 \\ ${ }^{1}$ Department of Hematology and ${ }^{2}$ Reproductive Medicine Center, The First Affiliated Hospital, \\ University of Science and Technology of China, Hefei, Anhui 230001, P.R. China
}

Received July 8, 2017; Accepted May 31, 2018

DOI: $10.3892 /$ ijmm.2018.3718

\begin{abstract}
Toll-like receptors (TLRs) are expressed in human bone marrow-derived mesenchymal stromal cells (BM-MSCs). The activation of TLRs is important in the proliferation, differentiation, migration and hematopoiesis-supporting functions of BM-MSCs. MicroRNAs (miRNAs) are involved in various biological functions by mediating mRNA degradation or inhibiting the translation of target genes. Our previous study confirmed that TLRs regulate the migration ability of BM-MSCs. It was also identified that multiple miRNAs were regulated by TLRs. In view of this, it was hypothesized that TLR-regulated miRNAs may be important in regulating the migration of BM-MSCs. The migration ability of BM-MSCs was evaluated following transfection of the cells with the mimics or antagonists of miRNA (miR)-27b, miR-146a, miR-155 and miR-154. miR-155 significantly inhibited cell migration. Myosin light chain kinase (MYLK) was identified as the direct target of miR-155 in BM-MSCs, which was further investigated using the luciferase reporter assay. However, miR-155 did not affect the expression of upstream proteins of the RhoA pathway controlling the activity of MYLK, suggesting that miR-155 directly suppressed the expression of MYLK without affecting the RhoA pathway. These results may facilitate the development and clinical use of BM-MSCs in terms of their migration.
\end{abstract}

Correspondence to: Dr Xingbing Wang, Department of Hematology, The First Affiliated Hospital, University of Science and Technology of China, 17 Lujiang Road, Hefei, Anhui 230001, P.R. China

E-mail: wangxingbing91@hotmail.com

Abbreviations: BM-MSCs, bone marrow derived-mesenchymal stromal cells; GO, Gene Ontology; miRNAs, microRNAs; MYLK, myosin light chain kinase; RT-qPCR, reverse transcription-quantitative polymerase chain reaction; TLRs, toll-like receptors

Key words: mesenchymal stem cells, migration, microRNAs, myosin light chain kinase

\section{Introduction}

MicroRNAs (miRNAs) are a class of small, non-coding RNAs (length, $22 \mathrm{nt}$ ), which regulate gene expression at the post-transcriptional level. miRNAs are involved in the regulation of the majority of important biological events, including differentiation, growth, proliferation, survival, signal transduction and immune response (1-3). However, the roles of miRNAs in the activation of human bone marrow-derived mesenchymal stromal cells (BM-MSCs) remain to be elucidated.

BM-MSCs are multipotent cells that differentiate into osteoblasts, adipocytes, chondrocytes and other tissue cells (4-7). MSCs not only support hematopoiesis and regulate immunity, but also specifically migrate to sites of tissue damage, chronic inflammation and tumors (8-11). For tumor therapy, MSCs can be used as a carrier for tumor resistance proteins, including interferon- $\alpha$ and $-\beta$, and specifically migrate to tumor sites to inhibit tumor cell growth $(12,13)$. MSCs can also home to bone marrow, repair damage in the hematopoietic microenvironment and promote hematopoietic reconstruction in patients following hematopoietic stem cell transplantation (14). MSCs have broad application prospects, however, the mechanism of MSC migration remains to be fully elucidated. An in-depth understanding of the mechanisms of MSC migration may enhance treatment efficiency by improving the ability of MSCs to migrate to target organs.

Toll-like receptors (TLRs) are an important class of protein molecules involved in innate immunity and acquired immunity (15). It has been demonstrated that TLRs are expressed in MSCs to modulate their proliferation, cytokine secretion, differentiation, hematopoiesis-supporting functions and immunosuppressive capacity $(16,17)$. Previous studies have suggested that the activation of TLR2 inhibits the migration of mice BM-MSCs (18). In our previous study, it was demonstrated that TLR2 was expressed on the surface of BM-MSCs and can suppress their migration (19). Notably, it is well established that TLRs induce multiple miRNAs, which in turn fine-tunes TLR-signaling responses at multiple levels. For example, miRNA (miR)-105, miR-146 and the let-7 miRNA family directly target the expression of TLR2 and TLR4 (20-23), whereas miR-155 and miR-146b target numerous TLR downstream signaling proteins $(24,25)$. Regulatory molecules, TLR-induced transcription factors and 
the final functional cytokines are also regulated by miRNAs, including miR-155 (26).

In our previous study, the activation of TLR2 induced the upregulation of miR-27b, miR-146a and miR-155, and the downregulation of miR-154 in BM-MSCs, indicating that they are TLR-responsive miRNAs (27). It was also found that TLR2 was expressed on the surface of BM-MSCs and that the activation of TLR2 decreased the migration ability of BM-MSCs $(18,19)$. These findings led to the present study testing the hypothesis that TLR2-responsive miRNAs are important in regulating BM-MSC migration ability. The results showed that miR-155 inhibited the cell migration of BM-MSCs and provided the first evidence, to the best of our knowledge, that miR-155 directly targets myosin light chain kinase (MYLK) in BM-MSCs.

\section{Materials and methods}

Cell culture. The present study was approved by the Medical Ethics Committee of Anhui Medical University (Anhui, China). Written informed consent was obtained from all participants. The BM-MSCs were isolated from fresh bone marrow of healthy donors. The isolation and culture of BM-MSCs have been described previously (21). In brief, following density gradient centrifugation for $20 \mathrm{~min}$ at $300 \mathrm{xg}$ at room temperature, mononuclear cells (MNCs) were cultured with high glucose concentration in Dulbecco's Modified Eagle Medium (HyClone; GE Healthcare Life Sciences, Logan, UT, USA) supplemented with $10 \%$ fetal calf serum (Gibco; Thermo Fisher Scientific, Inc., Waltham, MA, USA) in a $25 \mathrm{~cm}^{2}$ culture flask (Corning Incorporated, Corning, $\mathrm{NY}$, USA) at a concentration of $1 \times 10^{6} \mathrm{MNCs} / \mathrm{ml}$ at $37^{\circ} \mathrm{C}$ in an atmosphere of $5 \% \mathrm{CO}_{2}$. After $24 \mathrm{~h}$, the non-adherent cells were removed, and the adherent cells were cultured further. The medium was replaced twice each week until the cells were $\sim 90 \%$ confluent. The cells were then released by trypsin digestion and passaged into new culture flasks. Only MSCs in early passages (passage 3-5) were used. The BM-MSCs were analyzed by flow cytometry following staining with antibodies against CD90 (cat. no. 555595), CD14 (cat. no. 555397), CD29 (cat. no. 555443), CD34 (cat. no. 555823), CD166 (cat. no. 559263), CD44 (cat. no. 555478), CD31 (cat. no. 560983), CD45 (cat. no. 555482), CD13 (cat. no. 560998) and CD105 (cat. no. 561443) were all purchased from all: BD Biosciences, Franklin Lakes, NJ, USA).

Cell transfection. The miRNAs mimics, miRNAs inhibitor and MYLK small interfering (si)RNA were purchased from GenePharma Co., Ltd. (Shanghai, China). The sequences of the miRNAs were as follows: miR-27b mimics, 5'-AGAGCUUAG CUGAUUGGUGAAC-3'; miR-27b inhibitor, 5'-GUUCACCAA UCAGCUAAGCUCU-3'; miR-146a mimics, 5'-UGAGAACUG AAUUCCAUGGGUU-3'; miR-146a inhibitor, 5'-AACCCA UGGAAUUCAGUUCUCA-3'; miR-155 mimics, 5'-UUAAUG CUAAUCGUGAUAGGGU-3'; miR-155 inhibitor, 5'-ACCCCU AUCACGAUUAGCAUUAA-3'; miR-154 mimics, 5'-AAUCAU ACACGGUUGACAUAUU-3'; miR-154 inhibitor, 5'-AAUAGG UCAACCGUGUAUGAUU-3'; MYLK siRNA, 5'-GCCAAG AUGUUGUGAGCAATT-3'. At 1 day prior to transfection, the BM-MSCs were seeded in serum-free medium (Gibco; Thermo
Fisher Scientific, Inc.). The following day, $20 \mu \mathrm{mol} / 1 \mathrm{miRNA}$ was transfected using Lipofectamine 2000 (Invitrogen; Thermo Fisher Scientific, Inc.) according to the manufacturer's protocol. The cells were treated at $48 \mathrm{~h}$ post-transfection.

Reverse transcription-quantitative polymerase chain reaction (RT-qPCR) analysis. Total RNA was extracted from the cells using TRIzol reagent (Invitrogen; Thermo Fisher Scientific, Inc.). All RT reactions were performed using 1,000 ng of total RNA according to the following temperature protocol: $37^{\circ} \mathrm{C}$ for $60 \mathrm{~min}$ and $95^{\circ} \mathrm{C}$ for $5 \mathrm{~min}$. miRNA quantification was performed by RT-qPCR analysis using the Step One Real-Time PCR system (Applied Bio systems; Thermo Fisher Scientific, Inc.) and the SYBR premix Ex Taq II kit (Takara Biotechnology Co, Ltd., Dalian, China) according to the manufacturers' protocols. For measurement of the expression of all miRNA transcripts, U6 was used as the internal reference. Relative expression of miRNA was evaluated using the $2^{-\Delta \Delta \mathrm{Cq}}$ method (28). Thermo cycling conditions used for qPCR were as follows: $95^{\circ} \mathrm{C}$ for $10 \mathrm{~min}$; followed by 40 cycles of $95^{\circ} \mathrm{C}$ for $10 \mathrm{sec}$ and $60^{\circ} \mathrm{C}$ for $60 \mathrm{sec}$. The primer sequences were as follows: U6, forward 5'-GCTTCGGCAGCACATATA CTAAAAT-3' and reverse 5'-CGCTTCACGAATTTGCGT GTCAT-3'; miR-27b, forward 5'-GGGGAAGAGCTTAGC TGATTG-3' and reverse 5'-GTGCGTGTCGTGGAGTCG-3'; miR-146a, forward 5'-GGGTGAGAACTGAATTCC-3' and reverse 5'-TGCGTGTCGTGGAGTC-3'; miR-154, forward 5'-GGGGGAATCATACACGGTTG-3' and reverse 5'-GTG CGTGTCGTGGAGTCG-3'; miR-155, forward 5'-GGGGGT AATGCTAATCGTGAT-3' and reverse 5'-GTGCGTGTC GTGGAGTCG-3'.

Cell migration assay. A total of $2 \times 10^{4} \mathrm{BM}-\mathrm{MSCs}$ in $100 \mu \mathrm{l}$ culture medium (Hyclone; GE Healthcare Life Sciences, Logan, UT, USA) were seeded in the upper insert of a Transwell with an $8-\mathrm{mm}$ pore-size membrane (Corning Incorporated, Corning, NY, USA), and $600 \mu$ culture medium was added to the lower chamber. Following incubation for $24 \mathrm{~h}$ at $37^{\circ} \mathrm{C}$, each membrane was fixed with $4 \%$ paraformaldehyde (Cellchip Biotechnology Co., Ltd., Beijing, China), and MSCs on the membrane were stained with trypan blue (Sigma-Aldrich; Merck KGaA, Darmstadt, Germany). The numbers of migrated BM-MSCs were determined by counting the number of cells beneath the filter membrane in five fields at a high magnification under an inverted microscope. The experiments were performed with three replicates for each condition.

Target gene prediction and Gene Ontology (GO) analysis. Three online search algorithms, TargetScan version 6.2 (http://www.targetscan.org/vert_60/), miRanda (http://www. microrna.org/microrna/home.do), and Microcosm Targets version 5 (http://www.ebi.ac.uk/enright-srv/microcosm/htdocs/targets/v5/) were used to predict the target genes of miR-155. The overlapping sections that were identified by these on-line tools were considered to be the target genes. To confirm the predicted target genes, the genes were subjected to analysis by the Gene Ontology project (http://www.geneontology.org). The ontology covers three domains: Biological Process, Cellular Component and Molecular Function. Fisher's exact test is used to determine 

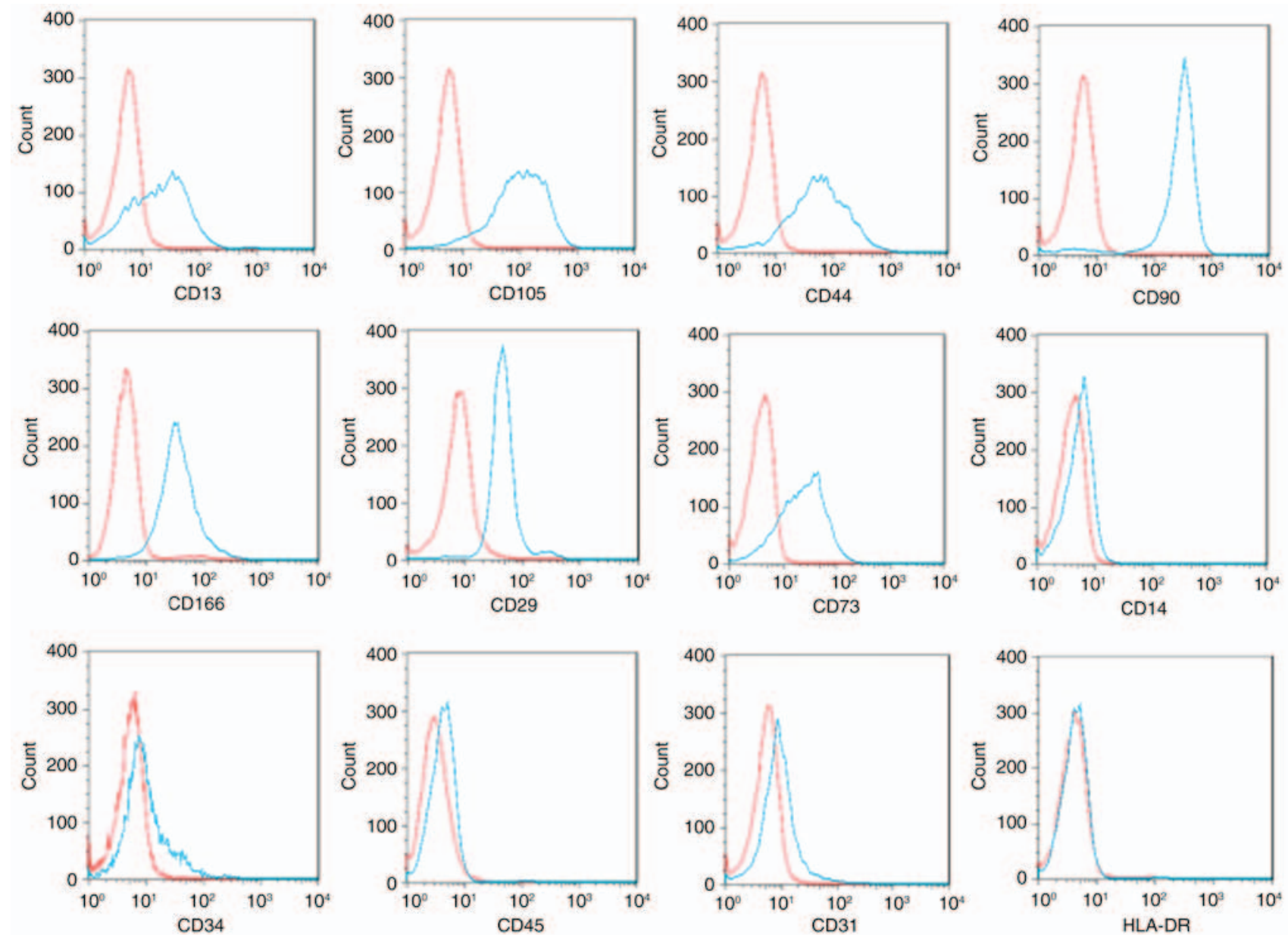

Figure 1. Immunophenotype of BM-MSCs. Representative results of the expression of lineage markers on proliferating adherent cells from healthy donor bone marrow, as assessed by flow cytometry. BM-MSCs, bone marrow derived-mesenchymal stromal cells.

whether there is more overlap between the differentially expressed (DE) list and the GO annotation list than would be expected by chance. The P-value denotes the significance of GO term enrichment in the DE genes. The lower the P-value, the more significant the GO term $(\mathrm{P} \leq 0.05$ is recommended).

Luciferase reporterassay. To confirm whether MYLK is a direct target of miR-155, a luciferase reporter assay was performed. 293T cells were seeded in a 48-well plate (Corning Incorporated) at $80 \%$ confluence and co-transfected with miR-155 mimics or inhibitors, and the psiCHECK-MYLK-3-untranslated region (UTR) or psiCHECK-MYLK-3-UTR-mutant (mut) vectors (GenePharma Co., Ltd.) using Lipofectamine 2000. Following incubation for $48 \mathrm{~h}$, the cells were collected and analyzed using a Dual-Luciferase assay kit (Promega Corporation, Madison, WI, USA). Each assay was performed with three replicates for each condition.

Western blot analysis. The cells were harvested $48 \mathrm{~h}$ following transfection with the miR-155 mimics or inhibitor. The cells were pelleted and lysed in lysing buffer $(5 \mu$ l protease inhibitor mixture, $5 \mu \mathrm{l}$ PMSF and $5 \mu \mathrm{l}$ phosphatase mixture). The lysates were centrifuged at $16,000 \mathrm{x}$ g for $15 \mathrm{~min}$ at $4^{\circ} \mathrm{C}$. Supernatants were subsequently collected and total protein concentration was determined using the Bio-Rad DC protein assay (Bio-Rad Laboratories, Inc., Hercules, CA, USA). An equal quantity of protein from each cell lysate $(20 \mu \mathrm{g})$ was separated by $4-12 \%$ sodium dodecyl sulfate-polyacrylamide gel electrophoresis and transferred onto a polyvinylidene fluoride membrane (Kangchen, Shanghai, China). The membranes were blocked with $5 \%$ bovine serum albumin (Sigma-Aldrich; Merck KGaA) followed by incubation overnight at $4^{\circ} \mathrm{C}$ with the following primary antibodies: Rabbit anti-human MYLK monoclonal antibody (cat. no. ab76092; 1:2,000 dilution; Abcam, Cambridge, MA, USA), rabbit anti-human RhoA monoclonal antibody (cat. no. ab187027; 1:1,000 dilution; Abcam), rabbit anti-human Rho-associated, coiled-coil containing protein kinase (Rock)1 monoclonal antibody (cat. no. ab134181; 1:2,000 dilution; Abcam), rabbit anti-human Rock2 monoclonal antibody (cat. no. ab125025; 1:10,000 dilution; Abcam) and rabbit anti-human $\beta$-actin monoclonal antibody (cat. no. ab150301; 1:10,000 dilution; Abcam). Incubation with the corresponding horseradish peroxidase-conjugated goat anti-rabbit secondary antibodies (cat. no. 32460; 1:2,000 dilution; Pierce; Thermo Fisher Scientific, Inc.) was performed for $1 \mathrm{~h}$ at $37^{\circ} \mathrm{C}$. Following three washes with TBS, the bound secondary antibody was visualized using enhanced chemiluminescence solution (Kangchen, Shanghai, China). Images were captured using the ImageJ system (version 1.50; National Institutes of Health, Bethesda, MD, USA). Densitometry was performed for comparison of western blot data (Alpha Innotech, San Leandro, CA, USA). 
Statistical analysis. Data were analyzed using SPSS 13.0 statistical software (SPSS, Inc., Chicago, IL, USA). Values are presented as the mean \pm standard deviation. For statistical comparisons, two-tailed Student's t-test (two-group) or one-way analysis of variance (multi-population) was applied as appropriate. $\mathrm{P}<0.05$ was considered to indicate a statistically significant difference.

\section{Results}

miR-155 inhibits the migration of BM-MSCs. In view of the significant inhibitory effect of TLR2 on the migration of BM-MSCs and the correlation of the TLR2 activation of miR-27b, miR-146a, miR-155 and miR-154, BM-MSCs were transfected with mimics or inhibitors of miR-27b, miR-146a, miR-155 and miR-154. The isolated BM-MSCs were positive for the markers CD90, CD105, CD166, CD29, CD44, $\mathrm{CD} 13$, and $\mathrm{CD} 73$, but negative for hematopoietic and endothelial lineage markers (CD14, CD34, CD31 and CD45) and HLA-DR (Fig. 1). RT-qPCR analysis was used to assess the expression of the four miRNAs in BM-MSCs following transfection. As expected, the expression levels of miRNAs were significantly upregulated following transfection with miRNA mimics, compared with levels in the negative control group. By contrast, the expression levels of miRNAs were downregulated following transfection with miRNA inhibitors (Fig. 2).

To examine the role of miRNA in the migration of BM-MSCs, cells transfected with miRNA mimics or inhibitor were cultured in Transwell chambers. The results of the Transwell assays showed that miR-155 mimics significantly suppressed the migration of BM-MSCs, whereas BM-MSC migration was enhanced following transfection with miR-155 inhibitors. miR-27b, miR-146a and miR-154 had no significant effect on the migration of BM-MSCs (Fig. 3A and B). The present study also evaluated the effects of the miRNA mimics/inhibitors on the cell viability of BM-MSCs in vitro. None of these compounds induced cell death of the BM-MSCs at $48 \mathrm{~h}$ post-transfection, assessed using the trypan blue staining method (data not shown).

MYLK is a direct target gene of miR-155 in BM-MSCs. To identify targets of miR-155 in BM-MSCs, bioinformatics analysis of miR-155 predicted target genes was performed using three online search algorithms, TargetScan version 6.2, miRanda and Microcosm Targets version 5, which identified 448, 3,387 and 930 potential targets, respectively, with an overlap of 74 genes. The overlapping sections that were identified by these online tools were considered to be the target genes. As shown in Fig. 4A, several genes were identified and subjected to GO analysis. The present study focused on genes associated with the Biological Process of cell movement. As a result, MYLK was predicted to be a target of miR-155 as the 3'-UTR of its mRNA contained a region with affinity for miR-155. To verify whether miR-155 directly targets MYLK, a luciferase reporter assay was performed. miR-155 was found to significantly inhibit the luciferase activity of the reporter vector containing the wild-type sequence of the MYLK 3'UTR targeted by miR-155, whereas the luciferase activity of the reporter vector containing a mutant sequence was not affected by miR-155 in the BM-MSCs (Fig. 4B; P<0.05).
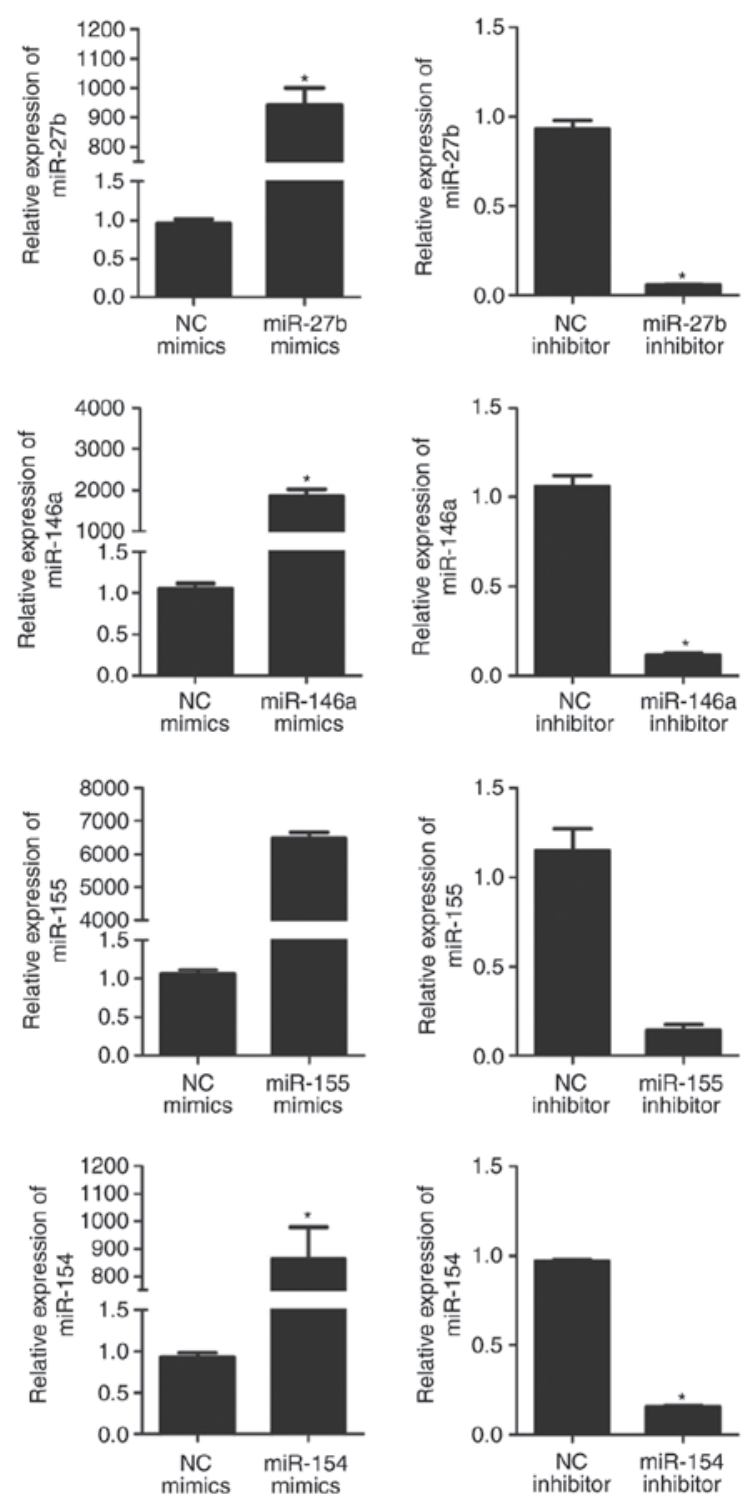

Figure 2. Relative expression of miRNA in BM-MSCs following transfection with miRNA mimics or inhibitor. Expression of miRNA was significantly upregulated following transfection with miRNA mimics and downregulated following transfection with miRNA inhibitors. The relative expression of miR-27b, miR-146a, miR-155 and miR-154 in BM-MSCs transfected with miRNA mimics NC were $0.96 \pm 0.05,1.06 \pm 0.06,1.06 \pm 0.06$ and $0.92 \pm 0.06$, respectively. The relative expression levels of miR-27b, miR-146a, miR-155 and miR-154 in BM-MSCs transfected with miRNA inhibitor NC were $0.93 \pm 0.05,1.06 \pm 0.07,1.15 \pm 1.12$ and $0.97 \pm 0.01$, respectively. The relative expression of miR-27b following transfection with miR-27b mimics and miR-27b inhibitor were 940.43 $\pm 58.84(\mathrm{P}<0.01)$ and $0.06 \pm 0.004(\mathrm{P}<0.01)$ compared with the respective control. The relative expression of miR-146a following transfection with miR-146a mimics and miR-146a inhibitor were $1,851.31 \pm 167.31(\mathrm{P}<0.01)$ and $0.11 \pm 0.01(\mathrm{P}<0.01)$ compared with the respective control. The relative expression of miR-155 following transfection with miR-155 mimics and miR-155 inhibitor were 6478.00 $\pm 170.15(\mathrm{P}<0.01)$ and $0.14 \pm 0.04(\mathrm{P}<0.01)$, compared with the respective control. The relative expression of miR-154 following transfection with miR-154 mimics and miR-154 inhibitor were 865.07 $\pm 114.10(\mathrm{P}<0.01)$ and $0.15 \pm 0.01(\mathrm{P}<0.01)$, compared with the respective control. Values are expressed as the mean \pm standard deviation. ${ }^{*} \mathrm{P}<0.05$, compared with the respective control. BM-MSCs, bone marrow derived-mesenchymal stromal cells; miR, microRNA; NC, negative control.

Furthermore, western blot analysis was performed to determine whether MYLK was decreased following transfection with miR-155 mimics. As shown in Fig. 5A-D, 

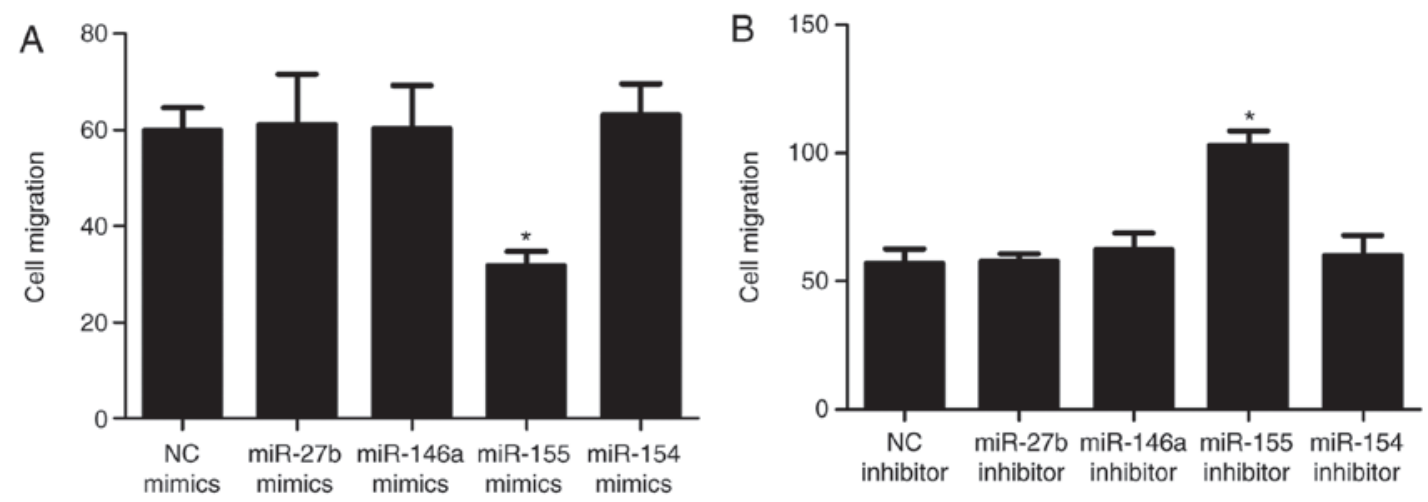

Figure 3. Role of miRNA in the migration of BM-MSCs. Migration following transfection with (A) miR mimics and (B) miR inhibitor. The numbers of migrated BM-MSCs following transfection with miRNA NC and miR-155 mimics were $60.00 \pm 4.58$ and $31.67 \pm 3.06$ ( $\mathrm{P}<0.01$ ), respectively; suggesting that miR-155 significantly suppressed the migration of BM-MSCs. Numbers of migrated BM-MSCs following transfection with miRNA inhibitor NC and miR-155 inhibitor were 57.00 \pm 5.57 and 103.00 $\pm 5.57(\mathrm{P}<0.01)$, respectively, showing BM-MSC migration was enhanced following transfection with miR-155 inhibitor and confirmed that miR-155 inhibits cell migration. Numbers of migrated BM-MSCs transfected with miR-27b mimics, miR-146a mimics and miR-154 mimics were 64.33 $\pm 5.03(\mathrm{P}>0.05), 60.33 \pm 8.96(\mathrm{P}>0.05)$ and 63.00 $\pm 6.56(\mathrm{P}>0.05)$ respectively, whereas the numbers of migrated BM-MSCs following transfection with miR-27b inhibitor, miR-146a inhibitor and miR-154 inhibitor were 58.00 $\pm 2.65(\mathrm{P}>0.05), 59.00 \pm 5.29(\mathrm{P}>0.05)$ and $58.00 \pm 4.00(\mathrm{P}>0.05)$, respectively, indicating miR-27b, miR-146a and miR-154 had no significant effect on migration. Values are expressed as the mean \pm standard deviation. ${ }^{*} \mathrm{P}<0.05$, compared with the respective control. BM-MSCs, bone marrow derived-mesenchymal stromal cells; miR, microRNA; NC, negative control.
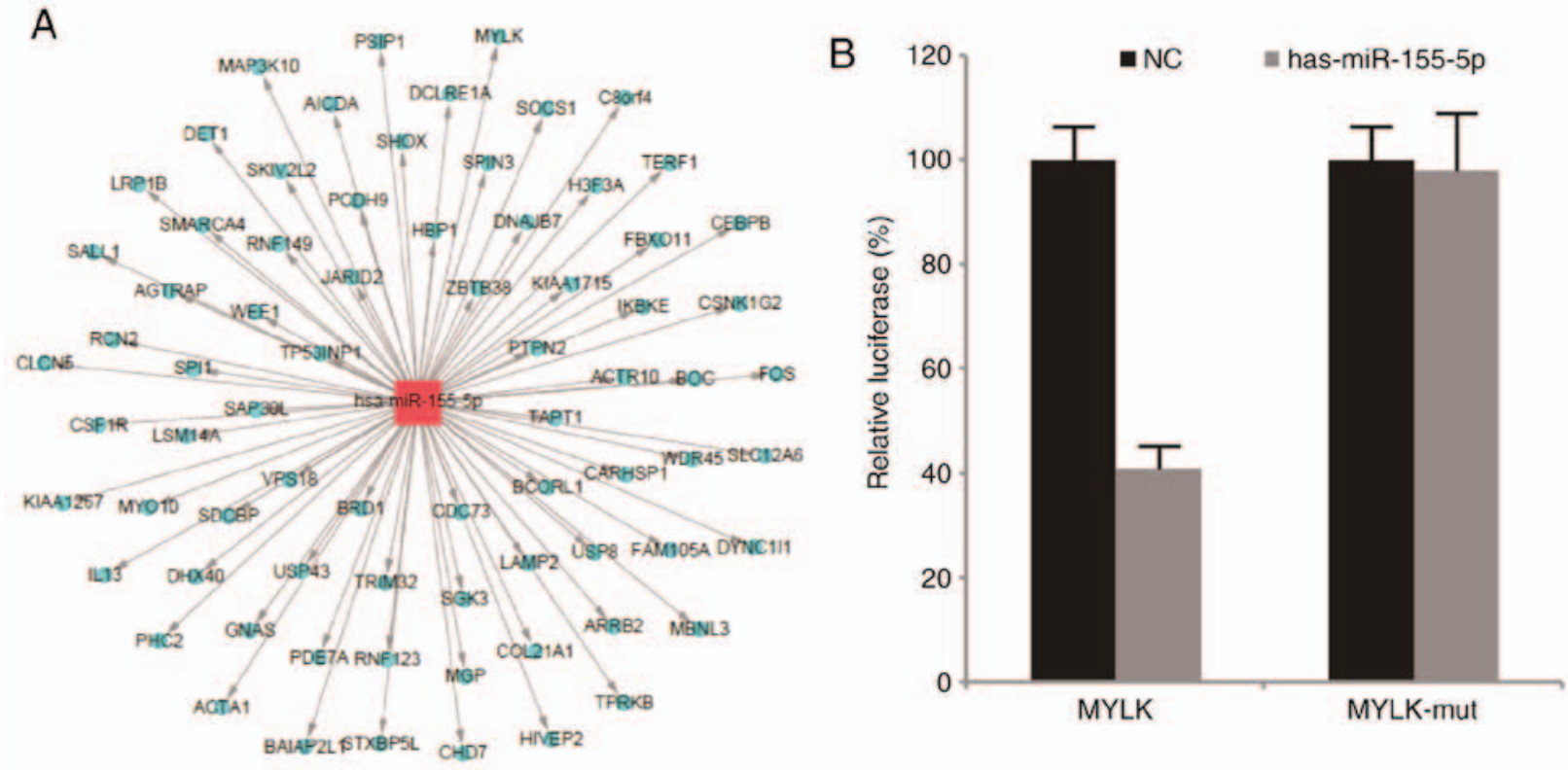

Figure 4. miR-155, target genes of miR-155, and their association. (A) Several target genes were identified by three online search algorithms. The light blue nodes indicate target genes and the red node indicates miR-155. (B) A luciferase reporter assay demonstrated that MYLK is a direct target of miR-155 in vitro. Relative luciferase of psiCHECK-MYLK in the control group and miR-155 transfection group were $1.00 \pm 0.07$ and $0.42 \pm 0.05$ ( $\mathrm{P}<0.05$ ) respectively, thus miR-155 significantly inhibited the luciferase activity of the reporter vector containing the wild-type sequence of the MYLK 3' untranslated region. Relative luciferase of psiCHECK-MYLK-mut in the control group and miR-155 transfection group was $1.00 \pm 0.10$ and $1.02 \pm 0.06$ (P>0.05) respectively, indicating luciferase activity of the reporter vector containing a mut sequence was not affected by miR-155 in BM-MSCs. BM-MSCs, bone marrow derived-mesenchymal stromal cells; miR, microRNA; NC, negative control; MYLK, myosin light chain kinase; mut, mutant.

the protein expression of MYLK was significantly downregulated following transfection with miR-155 mimics and upregulated following transfection with miR-155 inhibitors $(\mathrm{P}<0.05)$. In addition, the upstream proteins, RhoA, Rock1 and Rock2, were detected. As shown in Fig. 6A-D, no significant differences between the groups were observed. Therefore, it was hypothesized that miR-155 targeting MYLK does not affect the RhoA pathway. To further confirm the role of MYLK in regulating BM-MSC migration, siRNA was used to knock down MYLK in BM-MSCs. As shown in Fig. 7, the inhibition of MYLK by MYLK siRNA significantly suppressed the migration of BM-MSCs. Taken together, these results suggested that miR-155 inhibited BM-MSC migration via directly targeting MYLK.

\section{Discussion}

Human miR-155 is encoded by the miR-155 host gene and is involved in various physiological and pathological processes. miR-155 has been found to inhibit adipogenesis and immune 

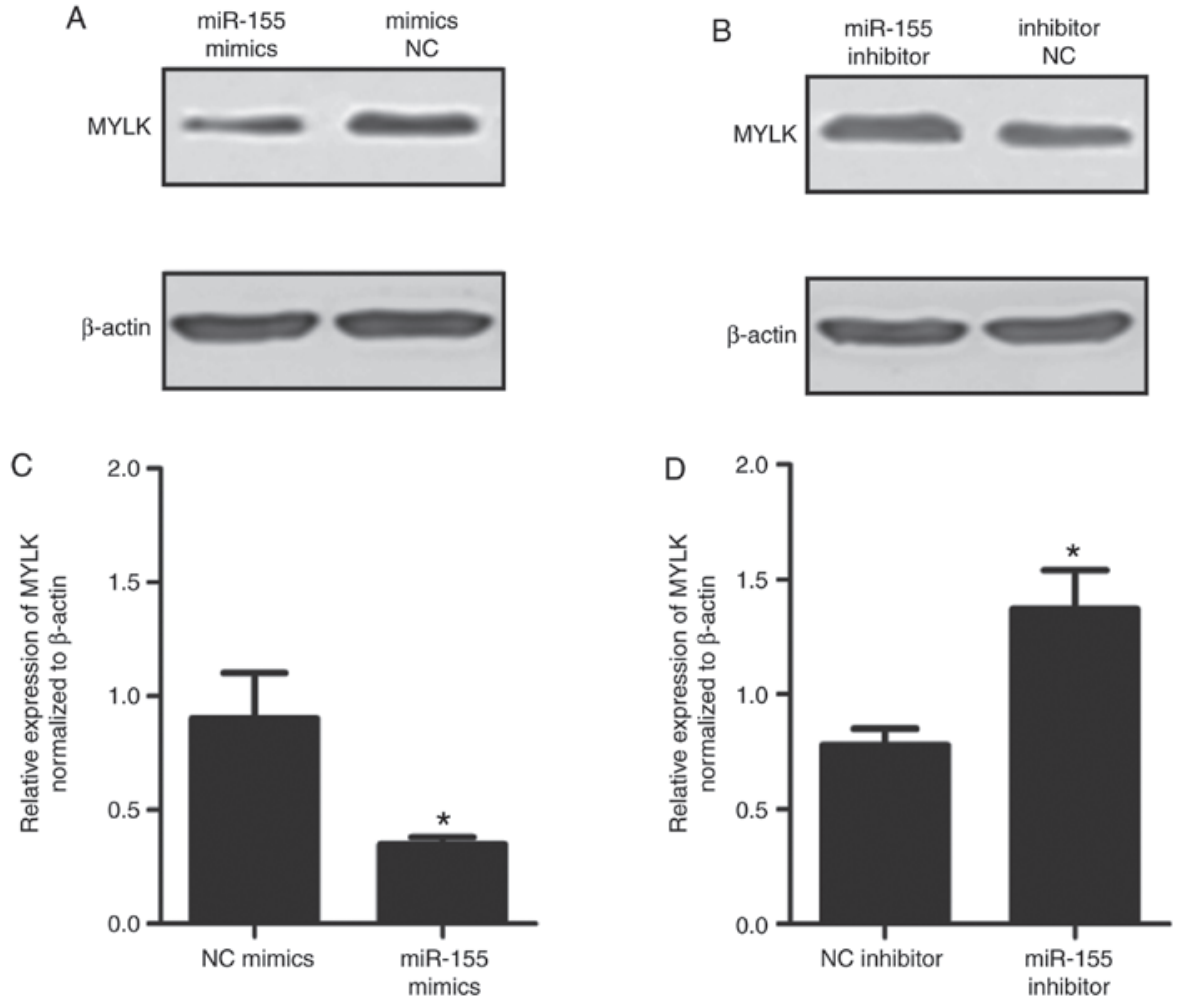

Figure 5. Protein expression of MYLK in BM-MSCs transfected with miR-155 inhibitors or mimics. (A) Relative protein expression of MYLK following transfection with miRNA mimic NC and miR-155 mimics were $0.90 \pm 0.20$ and $0.35 \pm 0.03$ ( $\mathrm{P}<0.05)$ respectively. (B) Relative protein expression of MYLK following transfection with miRNA inhibitor NC and miR-155 inhibitor were $0.78 \pm 0.07$ and $1.37 \pm 0.17(\mathrm{P}<0.05)$, respectively. Graphs show the quantified results for the (C) mimics and (D) inhibitor groups. miR-155 inhibited the protein expression of MYLK in the BM-MSCs. Values are expressed as the mean \pm standard deviation. "P<0.05, compared with the respective control. BM-MSCs, bone marrow derived-mesenchymal stromal cells; MYLK, myosin light chain kinase; miR, microRNA; NC, negative control.
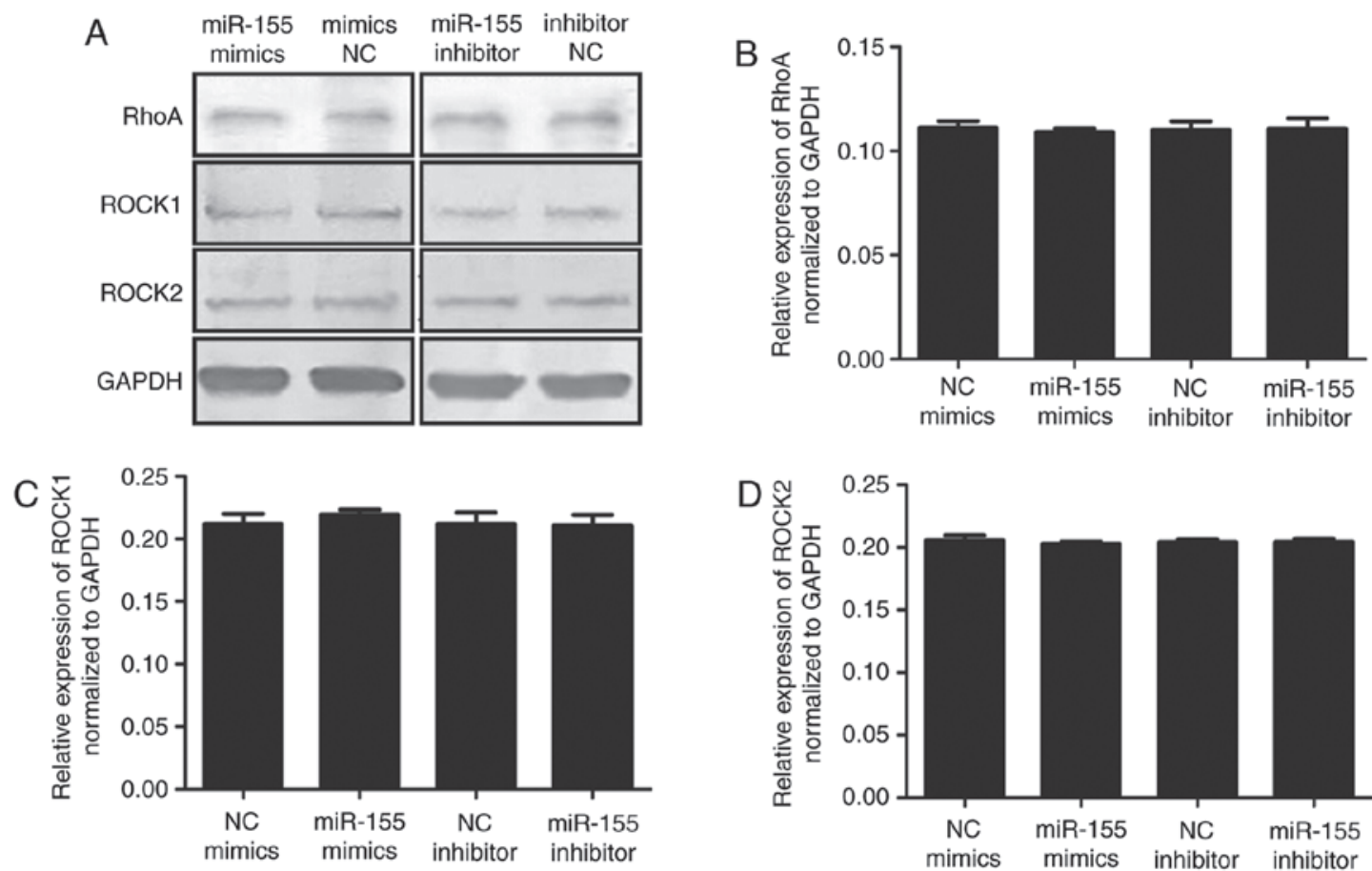

Figure 6. (A) Protein expression of RhoA, ROCK1 and ROCK2 in BM-MSCs transfected with miR-155 mimics or inhibitor. (B) The relative protein expression of RhoA following transfection with miRNA mimics NC, miR-155 mimics, miRNA inhibitor NC and miR-155 inhibitor were $0.111 \pm 0.003,0.108 \pm 0.001$ $(\mathrm{P}>0.05), 0.110 \pm 0.004$ and $0.110 \pm 0.005$ ( $P>0.05)$ respectively. (C) Relative protein expression of Rock1 following transfection with miRNA mimics NC, miR-155 mimics, miRNA inhibitor NC and miR-155 inhibitor were $0.212 \pm 0.008,0.220 \pm 0.003(\mathrm{P}>0.05), 0.212 \pm 0.009$ and $0.211 \pm 0.008$ ( $\mathrm{P}>0.05)$, respectively. (D) Relative protein expression of Rock2 following transfection with miRNA mimics NC, miR-155 mimics, miRNA inhibitor NC and miR-155 inhibitor were $0.205 \pm 0.004,0.203 \pm 0.002(\mathrm{P}>0.05), 0.204 \pm 0.002$ and $0.204 \pm 0.003(\mathrm{P}>0.05)$ respectively. BM-MSCs, bone marrow derived-mesenchymal stromal cells; Rock, Rho-associated, coiled-coil containing protein kinase; miR, microRNA; NC, negative control. 
regulation of MSCs, however, its effect on migration has not been reported (29,30). In the present study, the results showed that miR-155 significantly inhibited the migration of BM-MSCs. MYLK, a member of the immunoglobulin gene superfamily, encodes myosin light chain kinase, a calcium/calmodulin-dependent enzyme (31). MYLK phosphorylates the $\mathrm{N}$-terminus of the regulatory light chain of the molecular motor myosin II to produce contractility, which is involved in the migration of cells (32). Several studies have shown that MYLK is associated with cell migration. Weber et al found that MYLK is involved in the migration of endothelial cells. Their study confirmed that miR155 targets MYLK to inhibit cell migration (33). Miao et al found that MYLK-targeted siRNA decreased the expression of MYLK and cell migration of optic nerve head (ONH) astrocytes compared with control siRNA (34). This finding indicated that MYLK is a target in the inhibition of ONH astrocyte migration. Although MYLK is important in cell migration, its role in the migration of BM-MSCs has been unclear. Therefore, the present study is the first, to the best of our knowledge, to show that MYLK and related targets are involved in BM-MSC migration, and suggests an additional mechanism for the effect on BM-MSC migration.

The present study is the first, to the best of our knowledge, to demonstrate that miR-155 inhibits BM-MSC migration by targeting MYLK. Weber et al reported that miR-155 targeted MYLK to inhibit the migration of vascular endothelial cells (33). This issue is important in future investigations of miRNA, as it is essential for the application of a target gene of miRNA in one type of cell to other types of cells. In our previous study, it was found that the activation of TLR2 significantly inhibited the migration of BM-MSCs, and the expression of miR-155 was significantly upregulated following the activation of TLR2 $(19,27)$. The present study confirmed that miR-155 significantly inhibited the migration of BM-MSCs. Therefore, the evidence suggests that the mechanism of TLR2 inhibits BM-MSC migration, involving the upregulation of miR-155 and the inhibition of cell migration through miR-155 targeting MYLK. In addition, miR-27b, miR-146a and miR-154 were examined in the present study. The results showed that none of these three miRNAs had a marked effect on cell migration. Of note, a previous study reported that miR-146a can target stromal cell-derived factor-1 to inhibit BM-MSC migration (35). By comparison, this previous study involved counting the numbers of migrated BM-MSCs $12 \mathrm{~h}$ following the beginning of migration, whereas the $24 \mathrm{~h}$ time-point was used in the present study; this indicated a problem for cell migration in future investigations, as the different migration time may result in different results. In addition, miR-27b was found to inhibit the migration of mouse MSCs in a previous study (36). In the present study, miR-27b had no significant effect on the migration of human BM-MSCs, however, it is unclear whether this is due to differences between species, which requires further investigation.

In conclusion, the present study is the first, to the best of our knowledge, to show that miR-155 inhibited the migration of BM-MSCs by reducing the expression of MYLK. Through the identification of novel target genes of miR-155, the present study enhances current understanding of the mechanisms of BM-MSC migration. These findings may facilitate the devel-

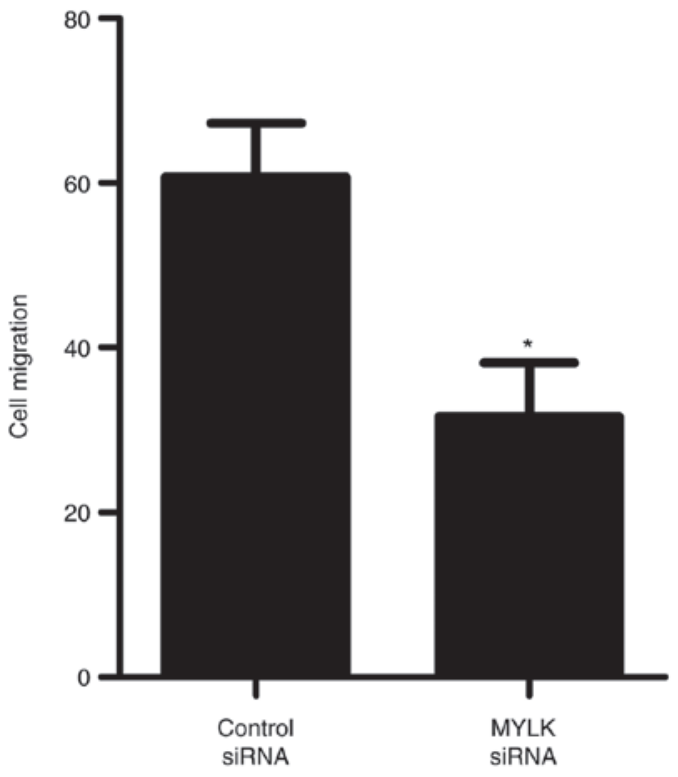

Figure 7. Numbers of migrated BM-MSCs following transfection with control siRNA and MYLK siRNA were $60.67 \pm 6.66$ and $31.67 \pm 6.51$, respectively. Compared with the control group, transfection with MYLK siRNA significantly inhibited cell migration $\left({ }^{*} \mathrm{P}<0.05\right)$. BM-MSCs, bone marrow derived-mesenchymal stromal cells; MYLK, myosin light chain kinase; siRNA, small interfering RNA

opment of the clinical use of BM-MSCs. Further investigation is required to identify additional target genes of miR-155 and to assess its suitability for use in clinical applications of BM-MSCs.

\section{Acknowledgements}

Not applicable.

\section{Funding}

This study was supported by the National Natural Science Foundation (grant no. 81270573), the Science and Technology key project of Anhui Province (grant no. 1604a0802071) and the Natural Science Foundation of Anhui Higher Education Institutions (grant no. KJ2012Z188).

\section{Availability of data and materials}

The datasets used during the present study are available from the corresponding author upon reasonable request.

\section{Authors' contributions}

XW designed the study, analyzed the data and contributed to the writing of the manuscript. XY, JJ and QZ performed the cell culture, cell transfection, cell migration assays, Luciferase reporter assays, reverse transcription-quantitative polymerase chain reaction analysis and contributed to the writing of the manuscript. BX performed Gene Ontology analysis. JW and JM performed cell culture and western blot analyses. XL analyzed the data. All authors read and approved the final manuscript. 


\section{Ethics approval and consent to participate}

The present study was approved by the Medical Ethics Committee of Anhui Medical University. Written informed consent was obtained from all participants.

\section{Patient consent for publication}

Not applicable.

\section{Competing interests}

The authors declare that they have no competing interests.

\section{References}

1. Yates LA, Norbury CJ and Gilbert RJ: The long and short of microRNA. Cell 153: 516-519, 2013.

2. Bartel DP: MicroRNAs: Target recognition and regulatory functions. Cell 136: 215-233, 2009.

3. Moreno-Moya JM, Vilella F and Simón C: MicroRNA: Key gene expression regulators. Fertil Steril 101: 1516-1523, 2014.

4. Pittenger MF, Mackay AM, Beck SC, Jaiswal RK, Douglas R, Mosca JD, Moorman MA, Simonetti DW, Craig S and Marshak DR: Multilineage potential of adult human mesenchymal stem cells. Science 284: 143-147, 1999.

5. Jiang Y, Jahagirdar BN, Reinhardt RL, Schwartz RE, Keene CD, Ortiz-Gonzalez XR, Reyes M, Lenvik T, Lund T, Blackstad M, et al: Pluripotency of mesenchymal stem cells derived from adult marrow. Nature 418: 41-49, 2002.

6. Charbord P: Bone marrow mesenchymal stem cells: Historical overview and concepts. Hum Gene Ther 21: 1045-1056, 2010.

7. Oreffo RO, Cooper C, Mason C and Clements M: Mesenchymal stem cells: Lineage, plasticity, and skeletal therapeutic potential Stem Cell Rev 1: 169-178, 2005.

8. Spaeth E, Klopp A, Dembinski J, Andreeff M and Marini F: Inflammation and tumor microenvironments: Defining the migratory itinerary of mesenchymal stem cells. Gene Ther 15: 730-738, 2008

9. Chamberlain G, Fox J, Ashton B and Middleton J: Concise review: Mesenchymal stem cells: Their phenotype, differentiation capacity, immunological features, and potential for homing. Stem Cells 25: 2739-2749, 2007.

10. Fox JM, Chamberlain G, Ashton BA and Middleton J: Recent advances into the understanding of mesenchymal stem cell trafficking. Br J Haematol 137: 491-502, 2007.

11. Warnke PH, Wiltfang J, Springer I, Acil Y, Bolte H, Kosmahl M, Russo PA, Sherry E, Lützen U, Wolfart S and Terheyden H: Man as living bioreactor: Fate of an exogenously prepared customized tissue-engineered mandible. Biomaterials 27: 3163-3167, 2006.

12. Ren C, Kumar S, Chanda D, Kallman L, Chen J, Mountz JD and Ponnazhagan S: Cancer gene therapy using mesenchymal stem cells expressing interferon-beta in a mouse prostate cancer lung metastasis model. Gene Ther 15: 1446-1453, 2008.

13. Studeny M, Marini FC, Champlin RE, Zompetta C, Fidler IJ and Andreeff $\mathrm{M}$ : Bone marrow derived mesenchymal stem cells as vehicles for interferon-beta delivery into tumors. Cancer Res 62: 3603-3608, 2002.

14. Pontikoglou C, Deschaseaux F, Sensebé L and Papadaki HA: Bone marrow mesenchymal stem cells: Biological properties and their role in hematopoiesis and hematopoietic stem cell transplantation. Stem Cell Rev 7: 569-589, 2011.

15. Kollmann TR, Levy O, Montgomery RR and Goriely S: Innate immune function by Toll-like receptors: Distinct responses in newborns and the elderly. Immunity 37: 771-783, 2012.

16. Shirjang S, Mansoori B, Solali S, Hagh MF and Shamsasenjan K: Toll-like receptors as a key regulator of mesenchymal stem cell function: An up-to-date review. Cell Immunol 315: 1-10, 2017.

17. Wang X, Cheng Q, Li L, Wang J, Xia L, Xu X and Sun Z: Toll-like receptors 2 and 4 mediate the capacity of mesenchymal stromal cells to support the proliferation and differentiation of CD34 ${ }^{+}$ cells. Exp Cell Res 318: 196-206, 2012.
18. Lei J, Wang Z, Hui D, Yu W, Zhou D, Xia W, Chen C, Zhang Q, Wang Z, Zhang Q and Xiang AP: Ligation of TLR2 and TLR4 on murine bone marrow-derived mesenchymal stem cells triggers differential effects on their immunosuppressive activity. Cell Immunol 271: 147-156, 2011.

19. Yang ZH, Wang XB, Wang J, Li LL and Zhu YX: Influence of TLR2 and TLR4 agonists on migration of human bone marrow mesenchymal stem cells. Zhongguo Shi Yan Xue Ye Xue Za Zhi 22: 183-186, 2014 (In Chinese).

20. Benakanakere MR, Li Q, Eskan MA, Singh AV, Zhao J, Galicia JC, Stathopoulou P, Knudsen TB and Kinane DF: Modulation of TLR2 protein expression by miR-105 in human oral keratinocytes. J Biol Chem 284: 23107-23115, 2009.

21. Quinn EM, Wang JH, O'Callaghan G and Redmond HP: MicroRNA-146a is upregulated by and negatively regulates TLR2 signaling. PLoS One 8: e62232, 2013.

22. O'Hara SP, Splinter PL, Gajdos GB, Trussoni CE, Fernandez-Zapico ME, Chen XM and LaRusso NF: NFkappaB p50-CCAAT/enhancer-binding protein beta (C/EBPbeta)-mediated transcriptional repression of microRNA let-7i following microbial infection. J Biol Chem 285: 216-225, 2010.

23. Yang K, He YS, Wang XQ, Lu L, Chen QJ, Liu J, Sun Z and Shen WF: MiR-146a inhibits oxidized low-density lipoprotein-induced lipid accumulation and inflammatory response via targeting toll-like receptor 4. FEBS Lett 585: 854-860, 2011.

24. Tili E, Michaille JJ, Cimino A, Costinean S, Dumitru CD, Adair B, Fabbri M, Alder H, Liu CG, Calin GA and Croce CM: Modulation of miR-155 and miR-125b levels following lipopolysaccharide/TNF-alpha stimulation and their possible roles in regulating the response to endotoxin shock. J Immunol 179: 5082-5089, 2007.

25. Curtale G, Mirolo M, Renzi TA, Rossato M, Bazzoni F and Locati M: Negative regulation of Toll-like receptor 4 signaling by IL-10-dependent microRNA-146b. Proc Natl Acad Sci USA 110: 11499-11504, 2013.

26. Ceppi M, Pereira PM, Dunand-Sauthier I, Barras E, Reith W, Santos MA and Pierre P: MicroRNA-155 modulates the interleukin-1 signaling pathway in activated human monocyte-derived dendritic cells. Proc Natl Acad Sci USA 106: 2735-2740, 2009.

27. Wang X, Zhu Y, Xu B, Wang J and Liu X: Identification of TLR2 and TLR4-induced microRNAs in human mesenchymal stem cells and their possible roles in regulating TLR signals. Mol Med Rep 13: 4969-4980, 2016.

28. Livak KJ and Schmittgen TD: Analysis of relative gene expression data using real-time quantitative PCR and the 2(-delta delta C(T)) method. Methods 25: 402-408, 2001.

29. Skårn M, Namløs HM, Noordhuis P, Wang MY, Meza-Zepeda LA and Myklebost O: Adipocyte differentiation of human bone marrow-derived stromal cells is modulated by microRNA-155, microRNA-221, and microRNA-222. Stem Cells Dev 21: 873-883, 2012.

30. Xu C, Ren G, Cao G, Chen Q, Shou P, Zheng C, Du L, Han X, Jiang M, Yang Q, et al: miR-155 regulates immune modulatory properties of mesenchymal stem cells by targeting TAK1-binding protein 2. J Biol Chem 288: 11074-11079, 2013.

31. Lazar V and Garcia JG: A single human myosin light chain kinase gene (MLCK; MYLK). Genomics 57: 256-267, 1999.

32. Kamm KE and Stull JT: Dedicated myosin light chain kinases with diverse cellular functions. J Biol Chem 276: 4527-4530, 2001.

33. Weber M, Kim S, Patterson N, Rooney K and Searles CD: MiRNA-155 targets myosin light chain kinase and modulates actin cytoskeleton organization in endothelial cells. Am J Physiol Heart Circ Physiol 306: H1192-H1203, 2014.

34. Miao H, Crabb AW, Hernandez MR and Lukas TJ: Modulation of factors affecting optic nerve head astrocyte migration. Invest Ophthalmol Vis Sci 51: 4096-4103, 2010.

35. Hsieh JY, Huang TS, Cheng SM, Lin WS, Tsai TN, Lee OK and Wang HW: miR-146a-5p circuitry uncouples cell proliferation and migration, but not differentiation, in human mesenchymal stem cells. Nucleic Acids Res 41: 9753-9763, 2013.

36. Lü MH, Li CZ, Hu CJ, Fan YH, Wang SM, Wu YY, Liang GP and Yang SM: microRNA-27b suppresses mouse MSC migration to the liver by targeting SDF-1 $\alpha$ in vitro. Biochem Biophys Res Commun 421: 389-395, 2012. 